Appl. Set-Valued Anal. Optim. 2 (2020), No. 1, pp. 1-17

Available online at http://asvao.biemdas.com

https://doi.org/10.23952/asvao.2.2020.1.01

\title{
PARALLEL COMPUTING PROXIMAL METHOD FOR NONSMOOTH CONVEX OPTIMIZATION WITH FIXED POINT CONSTRAINTS OF QUASI-NONEXPANSIVE MAPPINGS
}

\author{
KENGO SHIMIZU $^{1}$, KAZUHIRO HISHINUMA ${ }^{1}$, HIDEAKI IIDUKA ${ }^{2, *}$ \\ ${ }^{1}$ Computer Science Course, Graduate School of Science and Technology, Meiji University, \\ 1-1-1 Higashimita, Tama-ku, Kawasaki-shi, Kanagawa, 214-8571, Japan \\ ${ }^{2}$ Department of Computer Science, Meiji University, 1-1-1 Higashimita, Tama-ku, \\ Kawasaki-shi, Kanagawa, 214-8571, Japan
}

\begin{abstract}
We present a parallel computing proximal method for solving the problem of minimizing the sum of convex functions over the intersection of fixed point sets of quasi-nonexpansive mappings in a real Hilbert space. We also provide a convergence analysis of the method for constant and diminishing step sizes under certain assumptions as well as a convergence-rate analysis for a diminishing step size. Numerical comparisons show that the performance of the algorithm is comparable with existing subgradient methods.
\end{abstract}

Keywords. Fixed point; Nonsmooth convex optimization; Parallel computing; Proximal method; Quasinonexpansive mapping.

\section{INTRODUCTION}

In this paper, we consider the following problem [7, Problem 2.1] (see [3, 9, 10] for applications of Problem 1.1):

Problem 1.1. Let $H$ be a real Hilbert space. Suppose that

(A1) $Q_{i}: H \rightarrow H(i \in \mathscr{I}:=\{1,2, \ldots, I\})$ is quasi-firmly nonexpansive;

(A2) $f_{i}: H \rightarrow \mathbb{R}(i \in \mathscr{I})$ is convex and continuous with $\operatorname{dom}\left(f_{i}\right):=\left\{x \in H: f_{i}(x)<+\infty\right\}=$ $H$.

Then,

$$
\text { minimize } f(x):=\sum_{i \in \mathscr{I}} f_{i}(x) \text { subject to } x \in X:=\bigcap_{i \in \mathscr{I}} \operatorname{Fix}\left(Q_{i}\right),
$$

where one assumes that there exists a solution of Problem 1.1 (see Sections 2 and 4 for the details).

\footnotetext{
${ }^{*}$ Corresponding author.

E-mail addresses: kengo@cs.meiji.ac.jp (K. Shimizu), kaz@cs.meiji.ac.jp (K. Hishinuma), iiduka@cs.meiji.ac. jp (H. Iiduka).

Received October 31, 2019; Accepted January 26, 2020.
}

(C)2020 Applied Set-Valued Analysis and Optimization 
Algorithms for solving this problem were proposed in [7, 9]. Reference [7] proposed parallel and incremental subgradient methods for solving Problem 1.1 and provided convergence as well as convergence-rate analyses. Reference $[9,10]$ proposed stochastic fixed point optimization algorithms for solving a convex stochastic optimization problem that minimizes the expectation of $f_{i}$ s over Fix $\left(Q_{1}\right)$. The stochastic fixed point optimization algorithms can be applied to the classifier ensemble problem.

There are methods for solving Problem 1.1, where $Q_{i}$ is taken to be a nonexpansive mapping, which is a stronger assumption than a quasi-nonexpansive mapping. Subgradient methods were presented in $[4,5,6,11]$, while proximal methods were presented in $[8,16]$.

In this paper, we present a parallel method for solving Problem 1.1. The method is obtained by combining the parallel method in [7] with the proximal method in [8]. We also present a convergence analysis for a constant step size and a diminishing step size. The analysis shows that the proposed method with a small constant step size may approximate a solution to Problem 1.1 (Theorem 3.1) and that with a diminishing step size it converges to a solution under certain assumptions (Theorem 3.2). We also provide a convergence-rate analysis with a diminishing step size (Theorem 3.3). Finally, we numerically compare the proposed method with the existing subgradient methods.

This paper is organized as follows. Section 2 gives the mathematical preliminaries. Section 3 presents the parallel proximal method for solving Problem 1.1 and analyzes its convergence. Section 4 numerically compares the behaviors of the proposed method and the existing ones. Section 5 concludes the paper with a brief summary.

\section{Mathematical Preliminaries}

Let $H$ be a real Hilbert space with inner product $\langle\cdot, \cdot\rangle$ and its induced norm $\|\cdot\|$. We use the standard notation $\mathbb{N}$ for the natural numbers including zero and $\mathbb{R}^{N}$ for the $N$-dimensional Euclidean space.

2.1. Quasi-nonexpansivity and demiclosedness. The fixed point set of a mapping $Q: H \rightarrow H$ is denoted by

$$
\operatorname{Fix}(Q):=\{x \in H: Q(x)=x\} .
$$

$Q$ is said to be quasi-nonexpansive [2, Definition 4.1(iii)] if $\|Q(x)-y\| \leq\|x-y\|$ for all $x \in H$ and for all $y \in \operatorname{Fix}(Q)$. When a quasi-nonexpansive mapping has one fixed point, its fixed point set is closed and convex [2, Proposition 2.6]. $Q$ is said to be quasi-firmly nonexpansive [1, Section 3] if, for all $x \in H$ and for all $y \in \operatorname{Fix}(Q)$,

$$
\|Q(x)-y\|^{2}+\|(\operatorname{Id}-Q)(x)\|^{2} \leq\|x-y\|^{2},
$$

where $\operatorname{Id}(x):=x(x \in H)$. Any quasi-firmly nonexpansive mapping satisfies the quasi nonexpansivity condition. Moreover, $Q$ is quasi-firmly nonexpansive if and only if $R:=2 Q-\operatorname{Id}$ is quasi-nonexpansive [2, Proposition 4.2], which implies that $(1 / 2)(\operatorname{Id}+R)$ is quasi-firmly nonexpansive when $R$ is quasi-nonexpansive. Let $x, u \in H$ and $\left(x_{n}\right)_{n \in \mathbb{N}} \subset H$. Id $-Q$ is said to be demiclosed if a weak convergence of $\left(x_{n}\right)$ to $x$ and $\lim _{n \rightarrow+\infty}\left\|x_{n}-Q\left(x_{n}\right)-u\right\|=0$ imply $x-Q(x)=u$. Id $-Q$ is demiclosed when $Q$ is nonexpansive, i.e., $\|Q(x)-Q(y)\| \leq\|x-y\|$ $(x, y \in H)$ [2, Theorem 4.17]. The metric projection $P_{C}$ onto a nonempty, closed convex subset $C$ 
of $H$ is firmly nonexpansive, i.e., $\left\|P_{C}(x)-P_{C}(y)\right\|^{2}+\left\|\left(\operatorname{Id}-P_{C}\right)(x)-\left(\operatorname{Id}-P_{C}\right)(y)\right\|^{2} \leq\|x-y\|^{2}$ $(x, y \in H)$. Moreover, $\operatorname{Fix}\left(P_{C}\right)=C[2$, Proposition 4.8, (4.8)].

2.2. Convexity, proximal point, and subdifferentiability. A function $f: H \rightarrow \mathbb{R}$ is said to be convex if, for all $x, y \in H$ and for all $\alpha \in[0,1], f(\alpha x+(1-\alpha) y) \leq \alpha f(x)+(1-\alpha) f(y)$. A function $f$ is said to be strictly convex [2, Definition 8.6] if, for all $x, y \in H$ and for all $\alpha \in(0,1)$, $x \neq y$ implies $f(\alpha x+(1-\alpha) y)<\alpha f(x)+(1-\alpha) f(y) . f$ is strongly convex with constant $\beta$ [2, Definition 10.5] if there exists $\beta>0$ such that, for all $x, y \in H$ and for all $\alpha \in(0,1)$,

$$
f(\alpha x+(1-\alpha) y)+\frac{\beta \alpha(1-\alpha)}{2}\|x-y\|^{2} \leq \alpha f(x)+(1-\alpha) f(y) .
$$

Let $f: H \rightarrow(-\infty,+\infty]$ be proper, lower semicontinuous, and convex. Then, the proximity operator of $f$ [2, Definition 12.23], [14], denoted by $\operatorname{Prox}_{f}$, maps every $x \in H$ to the unique minimizer of $f(\cdot)+(1 / 2)\|x-\cdot\|^{2}$; i.e.,

$$
\left\{\operatorname{Prox}_{f}(x)\right\}=\underset{y \in H}{\operatorname{argmin}}\left[f(y)+\frac{1}{2}\|x-y\|^{2}\right](x \in H) .
$$

The uniqueness and existence of $\operatorname{Prox}_{f}(x)$ are guaranteed for all $x \in H$ [2, Definition 12.23], [13]. We call $\operatorname{Prox}_{f}(x)$ the proximal point of $f$ at $x$. Let $\operatorname{dom}(f):=\{x \in H: f(x)<+\infty\}$ be the domain of a function $f: H \rightarrow(-\infty,+\infty]$.

The subdifferential [2, Definition 16.1] of $f$ is defined by

$$
\partial f(x):=\{u \in H: f(y) \geq f(x)+\langle y-x, u\rangle(y \in H)\} \quad(x \in H) .
$$

We call $u \in \partial f(x)$ the subgradient of $f$ at $x$.

Proposition 2.1. [2, Propositions 12.26, 12.27, 12.28, and 16.14] Let $f: H \rightarrow(-\infty,+\infty]$ be proper, lower semicontinuous, and convex. Then, the following conclusions hold:

(i) Let $x, p \in H . p=\operatorname{Prox}_{f}(x)$ if and only if $x-p \in \partial f(p)$ (i.e., $\langle y-p, x-p\rangle+f(p) \leq f(y)$ for all $y \in H)$.

(ii) $\operatorname{Prox}_{f}$ is firmly nonexpansive with $\operatorname{Fix}\left(\operatorname{Prox}_{f}\right)=\operatorname{argmin}_{x \in H} f(x)$.

(iii) If $f$ is continuous at $x \in \operatorname{dom}(f), \partial f(x)$ is nonempty. Moreover, there exists $\delta>0$ such that $\partial f(B(x ; \delta))$ is bounded, where $B(x ; \delta)$ stands for a closed ball with center $x$ and radius $\delta$.

The following propositions will be used to prove the main theorems in this paper.

Proposition 2.2. [15, Lemma 3.1] Suppose that $\left(x_{n}\right)_{n \in \mathbb{N}} \subset H$ weakly converges to $\hat{x} \in H$ and $\bar{x} \neq \hat{x}$. Then, $\liminf _{n \rightarrow+\infty}\left\|x_{n}-\hat{x}\right\|<\liminf _{n \rightarrow+\infty}\left\|x_{n}-\bar{x}\right\|$.

Proposition 2.3. [2, Theorem 9.1] When $f: H \rightarrow \mathbb{R}$ is convex, $f$ is weakly lower semicontinuous if and only if $f$ is lower semicontinuous.

Proposition 2.4. $\left[12\right.$, Lemma 2.1] Let $\left(\Gamma_{n}\right)_{n \in \mathbb{N}} \subset \mathbb{R}$ and suppose that $\left(\Gamma_{n_{j}}\right)_{j \in \mathbb{N}}\left(\subset\left(\Gamma_{n}\right)_{n \in \mathbb{N}}\right)$ exists such that $\Gamma_{n_{j}}<\Gamma_{n_{j}+1}$ for all $j \in \mathbb{N}$. Define $(\tau(n))_{n \geq n_{0}} \subset \mathbb{N}$ by $\tau(n):=\max \left\{k \leq n: \Gamma_{k}<\right.$ $\left.\Gamma_{k+1}\right\}$ for some $n_{0} \in \mathbb{N}$. Then, $(\tau(n))_{n \geq n_{0}}$ is increasing and $\lim _{n \rightarrow+\infty} \tau(n)=+\infty$. Moreover, $\Gamma_{\tau(n)} \leq \Gamma_{\tau(n)+1}$ and $\Gamma_{n} \leq \Gamma_{\tau(n)+1}$ for all $n \geq n_{0}$. 


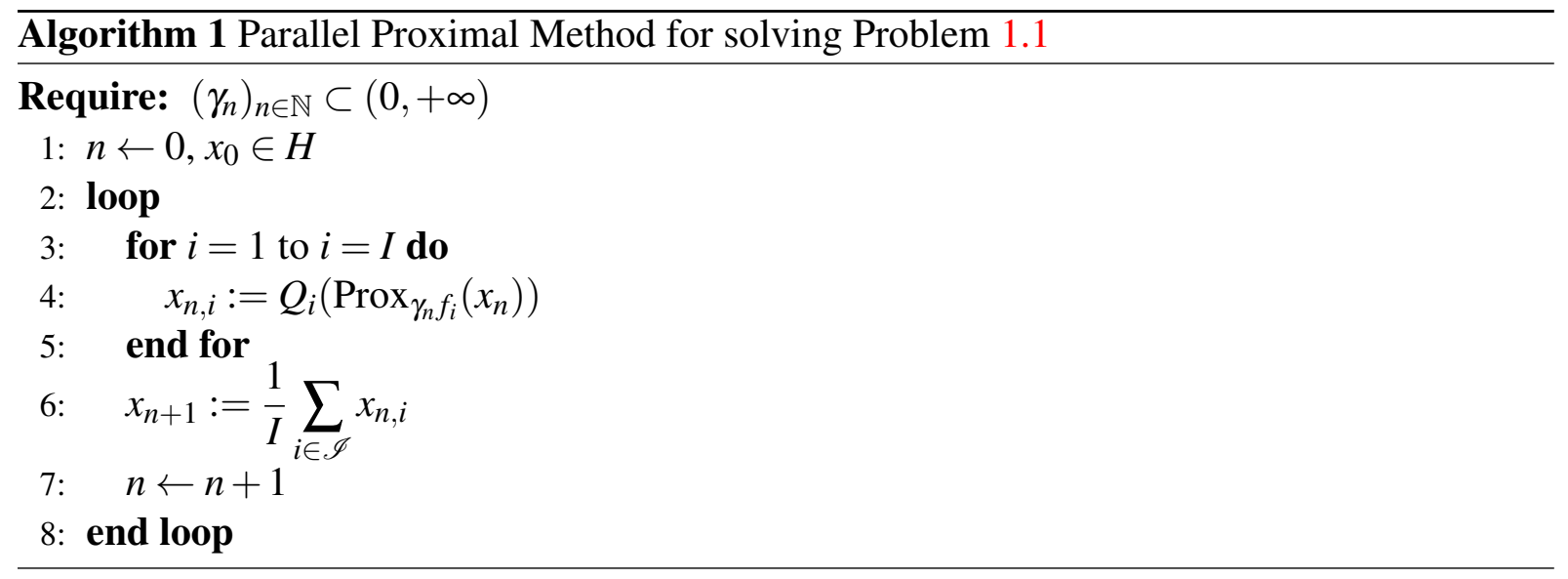

\section{The Parallel Proximal Method}

Algorithm 1 is the proposed algorithm for solving Problem 1.1.

Let us consider a network system with $I$ users and assume that user $i$ has its own private objective function $f_{i}$ and mapping $Q_{i}$ and tries to minimize $f_{i}$ over Fix $\left(Q_{i}\right)$. Moreover, let us assume that each user can communicate with other users. Then, at iteration $n$, each user can have $x_{n}$ in common. Since user $i$ has its own objective function $f_{i}$, it computes $y_{n, i}:=\operatorname{Prox} \gamma_{n} f_{i}\left(x_{n}\right)$. Moreover, user $i$ has its own constraint set Fix $\left(Q_{i}\right)$, with which it tries to find a fixed point of $Q_{i}$ by using $x_{n, i}:=Q_{i}\left(y_{n, i}\right)$. Since the users can communicate with each other, user $i$ can receive all $x_{n, i}$, and hence, user $i$ can compute $x_{n+1}:=(1 / I) \sum_{i \in \mathscr{I}} x_{n, i}$.

Let us compare Algorithm 1 with the existing parallel subgradient method [7, Algorithm 3.1] for solving Problem 1.1. The parallel subgradient method [7, Algorithm 3.1] is as follows:

$$
\begin{aligned}
& Q_{\alpha, i}:=\alpha \operatorname{Id}+(1-\alpha) Q_{i}, \\
& g_{n, i} \in \partial f_{i}\left(Q_{\alpha, i}\left(x_{n}\right)\right), \\
& x_{n, i}:=Q_{\alpha, i}\left(x_{n}\right)-\lambda_{n} g_{n, i}, \\
& x_{n+1}:=\frac{1}{I} \sum_{i \in \mathscr{I}} x_{n, i} .
\end{aligned}
$$

The difference between Algorithms 1 and (3.1) is the form of $x_{n, i}$, i.e., Algorithm 1 uses $x_{n, i}=$ $Q_{i}\left(\operatorname{Prox}_{\gamma_{n} f_{i}}\left(x_{n}\right)\right)$, while algorithm (3.1) uses $x_{n, i}:=Q_{\alpha, i}\left(x_{n}\right)-\lambda_{n} g_{n, i}$. Section 4 compares the behaviors of Algorithm 1 and algorithm (3.1) for concrete optimization problems.

First, we prove the following lemma.

Lemma 3.1. Suppose that (A1) and (A2) hold and define $y_{n, i}:=\operatorname{Prox}_{\gamma_{n} f_{i}}\left(x_{n}\right)$ for all $i \in \mathscr{I}$ and for all $n \in \mathbb{N}$. Then, Algorithm 1 satisfies that, for all $x \in X$ and for all $n \in \mathbb{N}$,

$$
\left\|x_{n+1}-x\right\|^{2} \leq\left\|x_{n}-x\right\|^{2}-\frac{1}{I} \sum_{i \in \mathscr{I}}\left\{\left\|x_{n}-y_{n, i}\right\|^{2}+\left\|x_{n, i}-y_{n, i}\right\|^{2}\right\}+\frac{2}{I} \gamma_{n} \sum_{i \in \mathscr{I}}\left(f_{i}(x)-f_{i}\left(y_{n, i}\right)\right) .
$$

Proof. Let $x \in X$ and $n \in \mathbb{N}$ be fixed arbitrarily. The definition of $y_{n, i}:=\operatorname{Prox} \gamma_{n} f_{i}\left(x_{n}\right)$ and Proposition 2.1(i) ensure that, for all $i \in \mathscr{I}$,

$$
\left\langle x-y_{n, i}, x_{n}-y_{n, i}\right\rangle \leq \gamma_{n}\left(f_{i}(x)-f_{i}\left(y_{n, i}\right)\right),
$$


which, together with $2\langle x, y\rangle=\|x\|^{2}+\|y\|^{2}-\|x-y\|^{2}(x, y \in H)$, implies that

$$
2 \gamma_{n}\left(f_{i}(x)-f_{i}\left(y_{n, i}\right)\right) \geq\left\|x-y_{n, i}\right\|^{2}+\left\|x_{n}-y_{n, i}\right\|^{2}-\left\|x-x_{n}\right\|^{2} .
$$

Accordingly, for all $i \in \mathscr{I}$,

$$
\left\|y_{n, i}-x\right\|^{2} \leq\left\|x_{n}-x\right\|^{2}-\left\|x_{n}-y_{n, i}\right\|^{2}+2 \gamma_{n}\left(f_{i}(x)-f_{i}\left(y_{n, i}\right)\right) .
$$

The definition of $x_{n, i}:=Q_{i}\left(y_{n, i}\right)$ and (A1) guarantee that, for all $i \in \mathscr{I}$,

$$
\left\|x_{n, i}-x\right\|^{2} \leq\left\|y_{n, i}-x\right\|^{2}-\left\|x_{n, i}-y_{n, i}\right\|^{2} .
$$

Hence, (3.2) and (3.3) imply that

$$
\left\|x_{n, i}-x\right\|^{2} \leq\left\|x_{n}-x\right\|^{2}-\left\|x_{n}-y_{n, i}\right\|^{2}-\left\|x_{n, i}-y_{n, i}\right\|^{2}+2 \gamma_{n}\left(f_{i}(x)-f_{i}\left(y_{n, i}\right)\right) .
$$

Summing the above inequality from $i=1$ to $i=I$ and the convexity of $\|\cdot\|^{2}$ ensure that

$$
\begin{aligned}
I\left\|x_{n+1}-x\right\|^{2} & \leq \sum_{i \in \mathscr{I}}\left\|x_{n, i}-x\right\|^{2} \\
& \leq I\left\|x_{n}-x\right\|^{2}-\sum_{i \in \mathscr{I}}\left\{\left\|x_{n}-y_{n, i}\right\|^{2}+\left\|x_{n, i}-y_{n, i}\right\|^{2}\right\}+2 \gamma_{n} \sum_{i \in \mathscr{I}}\left(f_{i}(x)-f_{i}\left(y_{n, i}\right)\right),
\end{aligned}
$$

which completes the proof.

The convergence analysis of Algorithm 1 depends on the following.

Assumpion 3.1. The sequence $\left(y_{n, i}\right)_{n \in \mathbb{N}}(i \in \mathscr{I})$ is bounded.

Assume that, for all $i \in \mathscr{I}, \operatorname{argmin}_{x \in H} f_{i}(x)\left(=\operatorname{Fix}\left(\operatorname{Prox}_{f_{i}}\right)\right) \neq \emptyset$ and $\operatorname{Fix}\left(Q_{i}\right)$ is bounded. Then, we can choose in advance of running the algorithm a bounded, closed convex set $C_{i}$ (e.g., $C_{i}$ is a closed ball with a large enough radius) satisfying $C_{i} \supset \operatorname{Fix}\left(Q_{i}\right)$. Accordingly, we can compute

$$
x_{n, i}:=P_{C_{i}}\left[Q_{i}\left(y_{n, i}\right)\right] \in C_{i}
$$

instead of $x_{n, i}$ in Algorithm 1. The boundedness of $C_{i}(i \in \mathscr{I})$ implies that $\left(x_{n, i}\right)_{n \in \mathbb{N}}(i \in \mathscr{I})$ is bounded. Accordingly, $\left(x_{n}\right)_{n \in \mathbb{N}}$ is also bounded. Moreover, Proposition 2.1(ii) ensures that, for all $i \in \mathscr{I}$, for all $n \in \mathbb{N}$, and for all $x \in \operatorname{Fix}\left(\operatorname{Prox}_{f_{i}}\right),\left\|y_{n, i}-x\right\| \leq\left\|x_{n}-x\right\|$. Hence, the boundedness of $\left(x_{n}\right)_{n \in \mathbb{N}}$ guarantees that $\left(y_{n, i}\right)_{n \in \mathbb{N}}(i \in \mathscr{I})$ is bounded. Hence, it can be assumed that $\left(x_{n, i}\right)_{n \in \mathbb{N}}(i \in \mathscr{I})$ in Algorithm 1 is as in (3.4) in place of Assumption 3.1.

We also have the following lemma.

Lemma 3.2. Suppose that (A1), (A2), and Assumption 3.1 hold. Then, $\left(x_{n, i}\right)_{n \in \mathbb{N}}(i \in \mathscr{I})$ and $\left(x_{n}\right)_{n \in \mathbb{N}}$ are bounded.

Proof. Assumption (A1) ensures that, for all $x \in X$, for all $i \in \mathscr{I}$, and for all $n \in \mathbb{N}$,

$$
\left\|x_{n, i}-x\right\| \leq\left\|y_{n, i}-x\right\|
$$

which, together with Assumption 3.1, implies that $\left(x_{n, i}\right)_{n \in \mathbb{N}}(i \in \mathscr{I})$ is bounded. Hence, the definition of $x_{n}$ implies that $\left(x_{n}\right)_{n \in \mathbb{N}}$ is also bounded. 
3.1. Constant step-size rule. The following is a convergence analysis of Algorithm 1 with a constant step size, which indicates that Algorithm 1 with a small constant step size may approximate a solution of Problem 1.1.

Theorem 3.1. Suppose that (A1), (A2), and Assumption 3.1 hold. Then, Algorithm 1 with $\gamma_{n}:=\gamma>0$ satisfies that

$$
\liminf _{n \rightarrow+\infty} \sum_{i \in \mathscr{I}}\left\|y_{n, i}-Q_{i}\left(y_{n, i}\right)\right\|^{2} \leq I M_{1} \gamma \quad \text { and } \quad \liminf _{n \rightarrow+\infty} \sum_{i \in \mathscr{I}} f_{i}\left(y_{n, i}\right) \leq f^{\star},
$$

where $M_{1}:=\sup \left\{(2 / I) \sum_{i \in \mathscr{I}}\left(f_{i}(x)-f_{i}\left(y_{n, i}\right)\right): n \in \mathbb{N}\right\}<+\infty$ for some $x \in X$ and $f^{\star}$ is the optimal value of Problem 1.1.

Proof. Let $x \in X$ be fixed arbitrarily. The definition of $\partial f_{i}(x)$ and the Cauchy-Schwarz inequality imply that, for all $i \in \mathscr{I}$, for all $n \in \mathbb{N}$, and for all $u_{i} \in \partial f_{i}(x)$,

$$
f_{i}(x)-f_{i}\left(y_{n, i}\right) \leq\left\langle x-y_{n, i}, u_{i}\right\rangle \leq\left\|y_{n, i}-x\right\|\left\|u_{i}\right\|,
$$

which, together with $\tilde{B}:=\max _{i \in \mathscr{I}} \sup \left\{\left\|y_{n, i}-x\right\|: n \in \mathbb{N}\right\}<+\infty$ (by Assumption 3.1), implies that

$$
M_{1}:=\sup \left\{\frac{2}{I} \sum_{i \in \mathscr{I}}\left(f_{i}(x)-f_{i}\left(y_{n, i}\right)\right): n \in \mathbb{N}\right\} \leq 2 \tilde{B} \max _{i \in \mathscr{I}}\left\|u_{i}\right\|<+\infty .
$$

We first show that

$$
\liminf _{n \rightarrow+\infty} \sum_{i \in \mathscr{I}} \underbrace{\left\{\left\|x_{n}-y_{n, i}\right\|^{2}+\left\|x_{n, i}-y_{n, i}\right\|^{2}\right\}}_{X_{n, i}} \leq I M_{1} \gamma .
$$

If (3.6) does not hold, there exists $\delta>0$ such that

$$
\liminf _{n \rightarrow+\infty} \sum_{i \in \mathscr{I}} X_{n, i}>I M_{1} \gamma+2 \delta \text {. }
$$

Accordingly, the property of the limit inferior of $\left(\sum_{i \in \mathscr{I}}\left\{\left\|x_{n}-y_{n, i}\right\|^{2}+\left\|x_{n, i}-y_{n, i}\right\|^{2}\right\}\right)_{n \in \mathbb{N}}$ ensures that $n_{0} \in \mathbb{N}$ exists such that, for all $n \geq n_{0}$,

$$
\sum_{i \in \mathscr{I}} X_{n, i}>I M_{1} \gamma+\delta
$$

Accordingly, Lemma 3.1 with $\gamma_{n}:=\gamma(n \in \mathbb{N})$ guarantees that, for all $n \geq n_{0}$,

$$
\begin{aligned}
\left\|x_{n+1}-x\right\|^{2} & \leq\left\|x_{n}-x\right\|^{2}-\frac{1}{I} \sum_{i \in \mathscr{I}} X_{n, i}+\frac{2}{I} \gamma \sum_{i \in \mathscr{I}}\left(f_{i}(x)-f_{i}\left(y_{n, i}\right)\right) \\
& <\left\|x_{n}-x\right\|^{2}-\frac{1}{I}\left(I M_{1} \gamma+\delta\right)+M_{1} \gamma \\
& =\left\|x_{n}-x\right\|^{2}-\frac{\delta}{I} \\
& <\left\|x_{n_{0}}-x\right\|^{2}-\frac{\delta}{I}\left(n+1-n_{0}\right) .
\end{aligned}
$$

The right side of the above inequality approaches minus infinity as $n$ diverges. Hence, we have a contradiction. This implies that (3.6) holds. Therefore,

$$
\liminf _{n \rightarrow+\infty} \sum_{i \in \mathscr{I}}\left\|y_{n, i}-x_{n, i}\right\|^{2}=\liminf _{n \rightarrow+\infty} \sum_{i \in \mathscr{I}}\left\|y_{n, i}-Q_{i}\left(y_{n, i}\right)\right\|^{2} \leq I M_{1} \gamma .
$$


Next, we show that

$$
\liminf _{n \rightarrow+\infty} \sum_{i \in \mathscr{I}} f_{i}\left(y_{n, i}\right) \leq f^{\star} .
$$

Assume that (3.8) does not hold. An argument similar to the one for obtaining (3.7) implies that there exist $\zeta>0$ and $m_{0} \in \mathbb{N}$ such that, for all $n \geq m_{0}$,

$$
\sum_{i \in \mathscr{I}} f_{i}\left(y_{n, i}\right)-f^{\star}>\zeta
$$

Lemma 3.1 thus ensures that, for all $n \geq m_{0}$ and for all $x^{\star} \in X^{\star}:=\left\{x^{\star} \in X: f\left(x^{\star}\right)=f^{\star}=\right.$ $\left.\inf _{x \in X} f(x)\right\}$,

$$
\begin{aligned}
\left\|x_{n+1}-x^{\star}\right\|^{2} & \leq\left\|x_{n}-x^{\star}\right\|^{2}+\frac{2}{I} \gamma\left(f^{\star}-\sum_{i \in \mathscr{I}} f_{i}\left(y_{n, i}\right)\right) \\
& <\left\|x_{n}-x^{\star}\right\|^{2}-\frac{2}{I} \gamma \zeta \\
& <\left\|x_{m_{0}}-x^{\star}\right\|^{2}-\frac{2}{I} \gamma \zeta\left(n+1-m_{0}\right),
\end{aligned}
$$

which is a contradiction. Accordingly, (3.8) holds. This completes the proof.

3.2. Diminishing step-size rule. The following is a convergence analysis of Algorithm 1 with a diminishing step size.

Theorem 3.2. Suppose that (A1), (A2), and Assumption 3.1 hold and $\operatorname{Id}-Q_{i}(i \in \mathscr{I})$ is demiclosed. ${ }^{*}$ Let $\left(x_{n}\right)_{n \in \mathbb{N}}$ be the sequence generated by Algorithm 1 with $\left(\gamma_{n}\right)_{n \in \mathbb{N}}$ satisfying $\lim _{n \rightarrow+\infty} \gamma_{n}=0$ and $\sum_{n=0}^{+\infty} \gamma_{n}=+\infty$. Then, there exists a subsequence of each of $\left(x_{n}\right)_{n \in \mathbb{N}}$, $\left(x_{n, i}\right)_{n \in \mathbb{N}}$, and $\left(y_{n, i}\right)_{n \in \mathbb{N}}(i \in \mathscr{I})$ that weakly converges to a solution of Problem 1.1. Moreover, $\left(x_{n}\right)_{n \in \mathbb{N}},\left(x_{n, i}\right)_{n \in \mathbb{N}}$, and $\left(y_{n, i}\right)_{n \in \mathbb{N}}(i \in \mathscr{I})$ strongly converge to a unique solution of Problem 1.1 if one of the following holds:

(i) One $f_{i}$ is strongly convex;

(ii) $H$ is finite-dimensional, and one $f_{i}$ is strictly convex.

Proof. We consider two cases.

Case 1: Suppose that there exists $m_{0} \in \mathbb{N}$ such that, for all $n \in \mathbb{N}$ and for all $x^{\star} \in X^{\star}, n \geq m_{0}$ implies $\left\|x_{n+1}-x^{\star}\right\| \leq\left\|x_{n}-x^{\star}\right\|$, where $X^{\star}:=\left\{x^{\star} \in X: f\left(x^{\star}\right)=f^{\star}=\inf _{x \in X} f(x)\right\}$. Then, there exists $c:=\lim _{n \rightarrow+\infty}\left\|x_{n}-x^{\star}\right\|$. Let $x^{\star} \in X^{\star}$ be fixed arbitrarily. Lemma 3.1, together with a discussion similar to that of (3.5), guarantees that there exists

$$
M_{2}:=\sup \left\{\frac{2}{I} \sum_{i \in \mathscr{I}}\left(f_{i}\left(x^{\star}\right)-f_{i}\left(y_{n, i}\right)\right): n \in \mathbb{N}\right\}<+\infty
$$

such that, for all $n \geq m_{0}$,

$$
\frac{1}{I} \sum_{i \in \mathscr{I}}\left\{\left\|x_{n}-y_{n, i}\right\|^{2}+\left\|x_{n, i}-y_{n, i}\right\|^{2}\right\} \leq\left\|x_{n}-x^{\star}\right\|^{2}-\left\|x_{n+1}-x^{\star}\right\|^{2}+M_{2} \gamma_{n} .
$$

* See Section 4 for an example in which $Q_{i}$ is quasi-firmly nonexpansive and $\mathrm{Id}-Q_{i}$ is demiclosed. 
Accordingly, the conditions $\lim _{n \rightarrow+\infty} \gamma_{n}=0$ and $c:=\lim _{n \rightarrow+\infty}\left\|x_{n}-x^{\star}\right\|$ mean that

$$
\lim _{n \rightarrow+\infty}\left\|x_{n}-y_{n, i}\right\|=0 \text { and } \lim _{n \rightarrow+\infty}\left\|x_{n, i}-y_{n, i}\right\|=0(i \in \mathscr{I}) \text {. }
$$

From Lemma 3.1, for all $x \in X$ and for all $k \in \mathbb{N}$, we have

$$
\frac{2}{I} \gamma_{k} \underbrace{\sum_{i \in \mathscr{I}}\left(f_{i}\left(y_{k, i}\right)-f_{i}(x)\right)}_{N_{k}(x)} \leq\left\|x_{k}-x\right\|^{2}-\left\|x_{k+1}-x\right\|^{2},
$$

which implies that, for all $n \in \mathbb{N}$ and for all $x \in X$,

$$
\frac{2}{I} \sum_{k=0}^{n} \gamma_{k} N_{k}(x) \leq\left\|x_{0}-x\right\|^{2}-\left\|x_{n+1}-x\right\|^{2} \leq\left\|x_{0}-x\right\|^{2} .
$$

Accordingly, for all $x \in X$,

$$
\frac{2}{I} \sum_{k=0}^{+\infty} \gamma_{k} N_{k}(x)<+\infty
$$

Here, we show that, for all $x \in X$,

$$
\liminf _{n \rightarrow+\infty} N_{n}(x) \leq 0
$$

Assume that (3.13) does not hold; i.e., there exists $x_{0} \in X$ such that $\liminf _{n \rightarrow+\infty} N_{n}\left(x_{0}\right)>0$. Then, $m_{1} \in \mathbb{N}$ and $\theta>0$ exist such that, for all $n \geq m_{1}, N_{n}\left(x_{0}\right) \geq \theta$. From (3.12) and $\sum_{n=0}^{+\infty} \gamma_{n}=$ $+\infty$, we have

$$
+\infty=\frac{2 \theta}{I} \sum_{k=m_{1}}^{+\infty} \gamma_{k} \leq \frac{2}{I} \sum_{k=m_{1}}^{+\infty} \gamma_{k} N_{k}\left(x_{0}\right)<+\infty,
$$

which is a contradiction. Hence, (3.13) holds, i.e., for all $x \in X$,

$$
\liminf _{n \rightarrow+\infty} \sum_{i \in \mathscr{I}} f_{i}\left(y_{n, i}\right) \leq \sum_{i \in \mathscr{I}} f_{i}(x)=: f(x)
$$

The definition of $u_{n, i} \in \partial f_{i}\left(x_{n}\right)$ and the Cauchy-Schwarz inequality ensure that, for all $i \in \mathscr{I}$ and for all $n \in \mathbb{N}$,

$$
f_{i}\left(x_{n}\right)-f_{i}\left(y_{n, i}\right) \leq\left\langle x_{n}-y_{n, i}, u_{n, i}\right\rangle \leq\left\|x_{n}-y_{n, i}\right\|\left\|u_{n, i}\right\| .
$$

Proposition 2.1(iii) and the boundedness of $\left(x_{n}\right)_{n \in \mathbb{N}}$ (see also Lemma 3.2) guarantee that there exists $B_{1}:=\max _{i \in \mathscr{I}} \sup \left\{\left\|u_{n, i}\right\|: n \in \mathbb{N}\right\}<+\infty$ such that, for all $n \in \mathbb{N}$,

$$
f\left(x_{n}\right)=\sum_{i \in \mathscr{I}} f_{i}\left(x_{n}\right) \leq B_{1} \sum_{i \in \mathscr{I}}\left\|x_{n}-y_{n, i}\right\|+\sum_{i \in \mathscr{I}} f_{i}\left(y_{n, i}\right) .
$$

Therefore, (3.10) and (3.14) lead to the finding that, for all $x \in X$,

$$
\liminf _{n \rightarrow+\infty} f\left(x_{n}\right) \leq B_{1} \lim _{n \rightarrow+\infty} \sum_{i \in \mathscr{I}}\left\|x_{n}-y_{n, i}\right\|+\liminf _{n \rightarrow+\infty} \sum_{i \in \mathscr{I}} f_{i}\left(y_{n, i}\right) \leq f(x) .
$$

Accordingly, a subsequence $\left(x_{n_{l}}\right)_{l \in \mathbb{N}}$ of $\left(x_{n}\right)_{n \in \mathbb{N}}$ exists such that, for all $x \in X$,

$$
\lim _{l \rightarrow+\infty} f\left(x_{n_{l}}\right)=\liminf _{n \rightarrow+\infty} f\left(x_{n}\right) \leq f(x) .
$$

Since $\left(x_{n_{l}}\right)_{l \in \mathbb{N}}$ is bounded (see also Lemma 3.2), there exists $\left(x_{n_{l}}\right)_{m \in \mathbb{N}}\left(\subset\left(x_{n_{l}}\right)_{l \in \mathbb{N}}\right)$ such that $\left(x_{n_{l m}}\right)_{m \in \mathbb{N}}$ weakly converges to $x_{*} \in H$. From (3.10), $\left(y_{n_{l_{m}}, i}\right)(i \in \mathscr{I})$ weakly converges to $x_{*}$. 
Hence, (3.10) and the demiclosedness of $\operatorname{Id}-Q_{i}$ ensure that $x_{*} \in \operatorname{Fix}\left(Q_{i}\right)(i \in \mathscr{I})$, i.e., $x_{*} \in X$. Proposition 2.3 ensures that the continuity and convexity of $f$ (by (A2)) imply that $f$ is weakly lower semicontinuous, which means that

$$
f\left(x_{*}\right) \leq \liminf _{m \rightarrow+\infty} f\left(x_{n_{l m}}\right) .
$$

Therefore, (3.16) leads to the finding that, for all $x \in X$,

$$
f\left(x_{*}\right) \leq \liminf _{m \rightarrow+\infty} f\left(x_{n_{l_{m}}}\right)=\lim _{m \rightarrow+\infty} f\left(x_{n_{l_{m}}}\right) \leq f(x),
$$

that is, $x_{*} \in X^{\star}$. Let us take another subsequence $\left(x_{n_{l_{k}}}\right)_{k \in \mathbb{N}}\left(\subset\left(x_{n_{l}}\right)_{l \in \mathbb{N}}\right)$ such that $\left(x_{n_{l_{k}}}\right)_{k \in \mathbb{N}}$ weakly converges to $x_{* *} \in H$. A discussion similar to the one for obtaining $x_{*} \in X^{\star}$ guarantees that $x_{* *} \in X^{\star}$. Here, it is proven that $x_{*}=x_{* *}$. Now, let us assume that $x_{*} \neq x_{* *}$. Then, the existence of $c:=\lim _{n \rightarrow+\infty}\left\|x_{n}-x^{\star}\right\|\left(x^{\star} \in X^{\star}\right)$ and Proposition 2.2 imply that

$$
\begin{aligned}
c & =\lim _{m \rightarrow+\infty}\left\|x_{n_{l_{m}}}-x_{*}\right\|<\lim _{m \rightarrow+\infty}\left\|x_{n_{l_{m}}}-x_{* *}\right\| \\
& =\lim _{n \rightarrow+\infty}\left\|x_{n}-x_{* *}\right\|=\lim _{k \rightarrow+\infty}\left\|x_{n_{l_{k}}}-x_{* *}\right\|<\lim _{k \rightarrow+\infty}\left\|x_{n_{l_{k}}}-x_{*}\right\| \\
& =c,
\end{aligned}
$$

which is a contradiction. Hence, $x_{*}=x_{* *}$. Accordingly, any subsequence of $\left(x_{n_{l}}\right)_{l \in \mathbb{N}}$ converges weakly to $x_{*} \in X^{\star}$; i.e., $\left(x_{n_{l}}\right)_{l \in \mathbb{N}}$ converges weakly to $x_{*} \in X^{\star}$. This means that $x_{*}$ is a weak cluster point of $\left(x_{n}\right)_{n \in \mathbb{N}}$ and belongs to $X^{\star}$. A discussion similar to the one for obtaining $x_{*}=x_{* *}$ guarantees that there is only one weak cluster point of $\left(x_{n}\right)_{n \in \mathbb{N}}$, so we can conclude that, in Case $1,\left(x_{n}\right)_{n \in \mathbb{N}}$ weakly converges to a point in $X^{\star}$.

Case 2: Suppose that, for all $m \in \mathbb{N}$, there exist $n \in \mathbb{N}$ and $x_{0}^{\star} \in X^{\star}$ such that $n \geq m$ and

$$
\left\|x_{n+1}-x_{0}^{\star}\right\|>\left\|x_{n}-x_{0}^{\star}\right\| \text {. }
$$

This implies that $\left(x_{n_{j}}\right)_{j \in \mathbb{N}}\left(\subset\left(x_{n}\right)_{n \in \mathbb{N}}\right)$ exists such that, for all $j \in \mathbb{N}$,

$$
\left\|x_{n_{j}+1}-x_{0}^{\star}\right\|>\left\|x_{n_{j}}-x_{0}^{\star}\right\|=: \Gamma_{n_{j}} .
$$

Proposition 2.4 thus guarantees that $m_{1} \in \mathbb{N}$ exists such that, for all $n \geq m_{1}, \Gamma_{\tau(n)} \leq \Gamma_{\tau(n)+1}$, where $\tau(n)$ is defined as in Proposition 2.4. From Lemma 3.1 (see also (3.9)), for all $n \geq m_{1}$, we have

$$
\begin{aligned}
\frac{1}{I} \sum_{i \in \mathscr{I}}\left\{\left\|x_{\tau(n)}-y_{\tau(n), i}\right\|^{2}+\left\|x_{\tau(n), i}-y_{\tau(n), i}\right\|^{2}\right\} & \leq \Gamma_{\tau(n)}^{2}-\Gamma_{\tau(n)+1}^{2}+\tilde{M}_{2} \gamma_{\tau(n)} \\
& \leq \tilde{M}_{2} \gamma_{\tau(n)},
\end{aligned}
$$

where

$$
\tilde{M}_{2}:=\sup \left\{\frac{2}{I} \sum_{i \in \mathscr{I}}\left(f_{i}\left(x^{\star}\right)-f_{i}\left(y_{\tau(n), i}\right)\right): n \in \mathbb{N}\right\}
$$

is finite by Assumption 3.1 (see also (3.5)). Hence, the condition $\lim _{n \rightarrow+\infty} \gamma_{\tau(n)}=0$ implies that

$$
\lim _{n \rightarrow+\infty}\left\|x_{\tau(n)}-y_{\tau(n), i}\right\|=0 \text { and } \lim _{n \rightarrow+\infty}\left\|x_{\tau(n), i}-y_{\tau(n), i}\right\|=0(i \in \mathscr{I}) .
$$

From (3.11), for all $n \geq m_{1}$,

$$
\frac{2}{I} \gamma_{\tau(n)} N_{\tau(n)}\left(x_{0}^{\star}\right) \leq \Gamma_{\tau(n)}^{2}-\Gamma_{\tau(n)+1}^{2} \leq 0,
$$


which, together with $\gamma_{\tau(n)} \geq 0\left(n \geq m_{1}\right)$, implies that $N_{\tau(n)}\left(x_{0}^{\star}\right) \leq 0$. Accordingly,

$$
\limsup _{n \rightarrow+\infty} \sum_{i \in \mathscr{I}} f_{i}\left(y_{\tau(n), i}\right) \leq f^{\star} .
$$

An argument, which is similar to the one for obtaining (3.15), together with (3.17), implies that

$$
\limsup _{n \rightarrow+\infty} f\left(x_{\tau(n)}\right) \leq f^{\star}
$$

Choose a subsequence $\left(x_{\tau\left(n_{k}\right)}\right)_{k \in \mathbb{N}}$ of $\left(x_{\tau(n)}\right)_{n \geq m_{1}}$ arbitrarily. Then,

$$
\limsup _{k \rightarrow+\infty} f\left(x_{\tau\left(n_{k}\right)}\right) \leq \limsup _{n \rightarrow+\infty} f\left(x_{\tau(n)}\right) \leq f^{\star} .
$$

The boundedness of $\left(x_{\tau\left(n_{k}\right)}\right)_{k \in \mathbb{N}}$ ensures that $\left(x_{\tau\left(n_{k_{l}}\right)}\right)_{l \in \mathbb{N}}\left(\subset\left(x_{\tau\left(n_{k}\right)}\right)_{k \in \mathbb{N}}\right)$ exists such that $\left(x_{\tau\left(n_{k_{l}}\right)}\right)_{l \in \mathbb{N}}$ weakly converges to $x_{\star} \in H$. Then, (3.17) and the demiclosedness of Id $-Q_{i}$ ensure that $x_{\star} \in X$. Moreover, Proposition 2.3 and (3.18) guarantee that

$$
f\left(x_{\star}\right) \leq \liminf _{l \rightarrow+\infty} f\left(x_{\tau\left(n_{k_{l}}\right)}\right) \leq \limsup _{l \rightarrow+\infty} f\left(x_{\tau\left(n_{k_{l}}\right)}\right) \leq f^{\star},
$$

that is, $x_{\star} \in X^{\star}$. Therefore, $\left(x_{\tau\left(n_{k_{l}}\right)}\right)_{l \in \mathbb{N}}$ weakly converges to $x_{\star} \in X^{\star}$. From Cases 1 and 2 , there exists a subsequence of $\left(x_{n}\right)_{n \in \mathbb{N}}$ that weakly converges to a point in $X^{\star}$.

Suppose that assumption (i) in Theorem 3.2 holds. The strong convexity of $f:=\sum_{i \in \mathscr{I}} f^{(i)}$ implies that $X^{\star}$ consists of one point, denoted by $x^{\star}$. In Case 1 , the strong convexity of $f$ guarantees that there exists $\beta>0$ such that, for all $\alpha \in(0,1)$ and for all $l \in \mathbb{N}$,

$$
(\beta / 2) \alpha(1-\alpha)\left\|x_{n_{l}}-x^{\star}\right\|^{2} \leq \alpha f\left(x_{n_{l}}\right)+(1-\alpha) f^{\star}-f\left(\alpha x_{n_{l}}+(1-\alpha) x^{\star}\right) .
$$

Accordingly, from the existence of $c:=\lim _{n \rightarrow+\infty}\left\|x_{n}-x^{\star}\right\|$ and (3.16), we have

$$
\begin{aligned}
\frac{\beta}{2} \alpha(1-\alpha) \lim _{l \rightarrow+\infty}\left\|x_{n_{l}}-x^{\star}\right\|^{2} \leq & \lim _{l \rightarrow+\infty}\left(\alpha f\left(x_{n_{l}}\right)+(1-\alpha) f^{\star}\right) \\
& +\limsup _{l \rightarrow+\infty}\left(-f\left(\alpha x_{n_{l}}+(1-\alpha) x^{\star}\right)\right) \\
\leq & f^{\star}-\liminf _{l \rightarrow+\infty} f\left(\alpha x_{n_{l}}+(1-\alpha) x^{\star}\right),
\end{aligned}
$$

which, together with the weak convergence of $\left(x_{n_{l}}\right)_{l \in \mathbb{N}}$ to $x^{\star}$ and Proposition 2.3, implies that

$$
\frac{\beta}{2} \alpha(1-\alpha) \lim _{l \rightarrow+\infty}\left\|x_{n_{l}}-x^{\star}\right\|^{2} \leq f^{\star}-f\left(\alpha x^{\star}+(1-\alpha) x^{\star}\right)=0 .
$$

Hence, $\left(x_{n_{l}}\right)_{l \in \mathbb{N}}$ strongly converges to $x^{\star}$. Therefore, from [2, Theorem 5.11], the whole sequence $\left(x_{n}\right)_{n \in \mathbb{N}}$ strongly converges to $x^{\star}$. From (3.10), $\left(x_{n, i}\right)_{n \in \mathbb{N}}$ and $\left(y_{n, i}\right)_{n \in \mathbb{N}}(i \in \mathscr{I})$ strongly converge to $x^{\star}$. In Case 2, the strong convexity of $f$ leads to the deduction that, for all $\alpha \in(0,1)$ and for all $l \in \mathbb{N}$,

$$
\begin{aligned}
\frac{\beta}{2} \alpha(1-\alpha) \limsup _{l \rightarrow+\infty}\left\|x_{\tau\left(n_{k_{l}}\right)}-x^{\star}\right\|^{2} \leq & \alpha \limsup _{l \rightarrow+\infty} f\left(x_{\tau\left(n_{k_{l}}\right)}\right)+(1-\alpha) f^{\star} \\
& -\liminf _{l \rightarrow+\infty} f\left(\alpha x_{\tau\left(n_{k_{l}}\right)}+(1-\alpha) x^{\star}\right) .
\end{aligned}
$$


The weak convergence of $\left(x_{\tau\left(n_{k_{l}}\right)}\right)_{l \in \mathbb{N}}$ to $x^{\star}$, the weakly lower semicontinuity of $f$ (by Proposition 2.3), and (3.18) imply that

$$
\frac{\beta}{2} \alpha(1-\alpha) \limsup _{l \rightarrow+\infty}\left\|x_{\tau\left(n_{k_{l}}\right)}-x^{\star}\right\|^{2} \leq f^{\star}-f\left(\alpha x^{\star}+(1-\alpha) x^{\star}\right)=0,
$$

which implies that $\left(x_{\tau\left(n_{k_{l}}\right)}\right)_{l \in \mathbb{N}}$ strongly converges to $x^{\star}$. When another subsequence $\left(x_{\tau\left(n_{k_{m}}\right)}\right)_{m \in \mathbb{N}}$ $\left(\subset\left(x_{\tau\left(n_{k}\right)}\right)_{k \in \mathbb{N}}\right)$ can be chosen, a discussion similar to the one for showing the weak convergence of $\left(x_{\tau\left(n_{k_{l}}\right)}\right)_{l \in \mathbb{N}}$ to a point in $X^{\star}$ guarantees that $\left(x_{\tau\left(n_{k_{m}}\right)}\right)_{m \in \mathbb{N}}$ also weakly converges to a point in $X^{\star}$. Furthermore, a discussion similar to the one for showing the strong convergence of $\left(x_{\tau\left(n_{k_{l}}\right)}\right)_{l \in \mathbb{N}}$ to $x^{\star}$ ensures that $\left(x_{\tau\left(n_{k_{m}}\right)}\right)_{m \in \mathbb{N}}$ strongly converges to the same $x^{\star}$. Hence, it is guaranteed that $\left(x_{\tau\left(n_{k}\right)}\right)_{k \in \mathbb{N}}$ strongly converges to $x^{\star}$. Since $\left(x_{\tau\left(n_{k}\right)}\right)_{k \in \mathbb{N}}$ is an arbitrary subsequence of $\left(x_{\tau(n)}\right)_{n \geq m_{1}},\left(x_{\tau(n)}\right)_{n \geq m_{1}}$ strongly converges to $x^{\star}$; i.e.,

$$
\lim _{n \rightarrow+\infty} \Gamma_{\tau(n)}=\lim _{n \rightarrow+\infty}\left\|x_{\tau(n)}-x^{\star}\right\|=0 .
$$

Accordingly, Proposition 2.4 ensures that

$$
\limsup _{n \rightarrow+\infty}\left\|x_{n}-x^{\star}\right\| \leq \limsup _{n \rightarrow+\infty} \Gamma_{\tau(n)+1}=0,
$$

which implies that, in Case 2, the whole sequence $\left(x_{n}\right)_{n \in \mathbb{N}}$ converges to $x^{\star}$. Moreover, Lemma 3.1 and $\lim _{n \rightarrow+\infty} \gamma_{n}=0$ imply that

$$
\lim _{n \rightarrow+\infty}\left\|x_{n}-y_{n, i}\right\|=\lim _{n \rightarrow+\infty}\left\|x_{n, i}-y_{n, i}\right\|=0,(i \in \mathscr{I}) .
$$

Therefore, $\left(x_{n, i}\right)_{n \in \mathbb{N}}$ and $\left(y_{n, i}\right)_{n \in \mathbb{N}}(i \in \mathscr{I})$ converge to $x^{\star}$.

Suppose that assumption (ii) in Theorem 3.2 holds. Let $x^{\star} \in X^{\star}$ be the unique solution to Problem 1.1. In Case 1, it is guaranteed that $\left(x_{n}\right)_{n \in \mathbb{N}}$ converges to $x^{\star} \in X^{\star}$. From (3.10), $\left(x_{n, i}\right)_{n \in \mathbb{N}}$ and $\left(y_{n, i}\right)_{n \in \mathbb{N}}(i \in \mathscr{I})$ strongly converge to $x^{\star}$. Moreover, in Case 2 , the convergence of $\left(x_{\tau\left(n_{k_{l}}\right)}\right)_{l \in \mathbb{N}}$ to $x^{\star}$ is guaranteed. A discussion similar to the one for showing the strong convergence of $\left(x_{\tau(n)}\right)_{n \geq m_{1}}$ to $x^{\star}$ ensures that $\left(x_{\tau(n)}\right)_{n \geq m_{1}}$ converges to $x^{\star} \in X^{\star}$. Proposition 2.4 thus guarantees that the whole sequence $\left(x_{n}\right)_{n \in \mathbb{N}}$ converges to $x^{\star}$. Lemma 3.1 and $\lim _{n \rightarrow+\infty} \gamma_{n}=$ 0 imply that

$$
\lim _{n \rightarrow+\infty}\left\|x_{n}-y_{n, i}\right\|=\lim _{n \rightarrow+\infty}\left\|x_{n, i}-y_{n, i}\right\|=0,(i \in \mathscr{I}) .
$$

Therefore, $\left(x_{n, i}\right)_{n \in \mathbb{N}}$ and $\left(y_{n, i}\right)_{n \in \mathbb{N}}(i \in \mathscr{I})$ converge to $x^{\star}$. This completes the proof.

The following is a convergence-rate analysis of Algorithm 1 with a diminishing step size.

Theorem 3.3. Suppose that the assumptions in Theorem 3.1 hold and a monotone decreasing sequence $\left(\gamma_{n}\right)_{n \in \mathbb{N}}$ satisfies $\lim _{n \rightarrow+\infty} \gamma_{n}=0, \lim _{n \rightarrow+\infty}\left(n \gamma_{n}\right)^{-1}=0, \sum_{n=0}^{+\infty} \gamma_{n}=+\infty$, and $\lim _{n \rightarrow+\infty} n^{-1}$ $\sum_{k=0}^{n-1} \gamma_{k}=0$. Then, Algorithm 1 satisfies that, for all $n \geq 1$,

$$
\sum_{i \in \mathscr{I}}\left(\frac{1}{n} \sum_{k=0}^{n-1}\left\|y_{k, i}-Q_{i}\left(y_{k, i}\right)\right\|^{2}\right) \leq \frac{I\left\|x_{0}-x\right\|^{2}}{n}+\frac{\tilde{M}_{1}}{n} \sum_{k=0}^{n-1} \gamma_{k},
$$

and

$$
\sum_{i \in \mathscr{I}} f_{i}\left(\frac{1}{n} \sum_{k=0}^{n-1} y_{k, i}\right) \leq f^{\star}+\frac{I B}{2 n \gamma_{n}}
$$


where $x^{\star}$ is a solution of Problem 1.1,

$$
\tilde{M}_{1}:=\sup \left\{2 \sum_{i \in \mathscr{I}}\left(f_{i}\left(x^{\star}\right)-f_{i}\left(y_{n, i}\right)\right): n \in \mathbb{N}\right\}<+\infty,
$$

and

$$
B:=\sup \left\{\left\|x_{n}-x^{\star}\right\|^{2}: n \in \mathbb{N}\right\}<+\infty .
$$

Proof. Let $x^{\star} \in X^{\star}$. Lemma 3.1 implies that, for all $n \geq 1$,

$$
\frac{1}{I} \sum_{i \in \mathscr{I}} \sum_{k=0}^{n-1}\left\{\left\|x_{k}-y_{k, i}\right\|^{2}+\left\|x_{k, i}-y_{k, i}\right\|^{2}\right\} \leq\left\|x_{0}-x\right\|^{2}+\frac{\tilde{M}_{1}}{I} \sum_{k=0}^{n-1} \gamma_{k},
$$

which in turn implies that

$$
\begin{aligned}
\sum_{i \in \mathscr{I}}\left(\frac{1}{n} \sum_{k=0}^{n-1}\left\|x_{k, i}-y_{k, i}\right\|^{2}\right) & \leq \frac{1}{n} \sum_{i \in \mathscr{I}} \sum_{k=0}^{n-1}\left\{\left\|x_{k}-y_{k, i}\right\|^{2}+\left\|x_{k, i}-y_{k, i}\right\|^{2}\right\} \\
& \leq \frac{I\left\|x_{0}-x\right\|^{2}}{n}+\frac{\tilde{M}_{1}}{n} \sum_{k=0}^{n-1} \gamma_{k} .
\end{aligned}
$$

Lemma 3.1 indicates that, for all $k \in \mathbb{N}$,

$$
\sum_{i \in \mathscr{I}} f_{i}\left(y_{k, i}\right)-f^{\star} \leq \frac{I}{2 \gamma_{k}}\left\{\left\|x_{k}-x^{\star}\right\|^{2}-\left\|x_{k+1}-x^{\star}\right\|^{2}\right\} .
$$

Summing the above inequality from $k=0$ to $k=n-1$ implies that, for all $n \geq 1$,

$$
\frac{1}{n} \sum_{k=0}^{n-1} \sum_{i \in \mathscr{I}} f_{i}\left(y_{k, i}\right)-f^{\star} \leq \frac{I}{2 n} \underbrace{\sum_{k=0}^{n-1} \frac{1}{\gamma_{k}}\left\{\left\|x_{k}-x^{\star}\right\|^{2}-\left\|x_{k+1}-x^{\star}\right\|^{2}\right\}}_{X_{n}} .
$$

The definition of $X_{n}$ means that

$$
X_{n}=\frac{\left\|x_{0}-x^{\star}\right\|}{\gamma_{0}}+\sum_{k=1}^{n-1}\left\{\frac{\left\|x_{k}-x^{\star}\right\|^{2}}{\gamma_{k}}-\frac{\left\|x_{k}-x^{\star}\right\|^{2}}{\gamma_{k-1}}\right\}-\frac{\left\|x_{n}-x^{\star}\right\|^{2}}{\gamma_{n-1}},
$$

which, together with $\gamma_{n} \leq \gamma_{n-1}(n \geq 1)$ and $B:=\sup \left\{\left\|x_{n}-x^{\star}\right\|^{2}: n \in \mathbb{N}\right\}<+\infty$ (by Lemma 3.2), implies that

$$
X_{n} \leq \frac{B}{\gamma_{0}}+B \sum_{k=1}^{n-1}\left(\frac{1}{\gamma_{k}}-\frac{1}{\gamma_{k-1}}\right)=\frac{B}{\gamma_{n-1}} \leq \frac{B}{\gamma_{n}} .
$$

The convexity of $f_{i}$ thus ensures that, for all $n \geq 1$,

$$
\sum_{i \in \mathscr{I}} f_{i}\left(\frac{1}{n} \sum_{k=0}^{n-1} y_{k, i}\right)-f^{\star} \leq \frac{I B}{2 n \gamma_{n}},
$$

which completes the proof. 
Let us consider the rate of convergence of Algorithm 1 with $\gamma_{n}:=n^{-1 / 2}(n \geq 1)$. The step size $\left(\gamma_{n}\right)_{n \geq 1}$ is monotone decreasing and satisfies $\lim _{n \rightarrow+\infty} \gamma_{n}=0, \lim _{n \rightarrow+\infty}\left(n \gamma_{n}\right)^{-1}=0$, and $\sum_{n=0}^{+\infty} \gamma_{n}=+\infty$. Moreover, the Cauchy-Schwarz inequality and $\sum_{k=0}^{n-1} k^{-1} \leq 1+\ln n$ mean that

$$
\frac{1}{n} \sum_{k=0}^{n-1} \frac{1}{\sqrt{k}} \leq \frac{\sqrt{n}}{n} \sqrt{\sum_{k=0}^{n-1} \frac{1}{k}} \leq \sqrt{\frac{1+\ln n}{n}}
$$

which implies that

$$
\lim _{n \rightarrow+\infty} \frac{1}{n} \sum_{k=0}^{n-1} \gamma_{k}=0
$$

Theorem 3.3 indicates that Algorithm 1 with $\gamma_{n}:=n^{-1 / 2}$ satisfies that, for all $n \geq 1$,

$$
\sum_{i \in \mathscr{I}}\left(\frac{1}{n} \sum_{k=0}^{n-1}\left\|y_{k, i}-Q_{i}\left(y_{k, i}\right)\right\|^{2}\right)=\mathscr{O}\left(\sqrt{\frac{1+\ln n}{n}}\right)
$$

and

$$
\sum_{i \in \mathscr{I}} f_{i}\left(\frac{1}{n} \sum_{k=0}^{n-1} y_{k, i}\right) \leq f^{\star}+\frac{I B}{2 \sqrt{n}},
$$

where $\mathscr{O}$ stands for the Landau notation (see [10] for a convergence rate analysis of stochastic approximation methods).

\section{NUMERICAL COMPARISONS}

Let us compare the performance of Algorithm 1 with the one of the existing parallel subgradient method (PSM) [7, Algorithm 3.1] (see (3.1)) and incremental subgradient method (ISM) [7, Algorithm 4.1] for the following problem (see also [7, Problem 5.1]): Let $a_{i, j}>0, b_{i, j}, d_{i} \in \mathbb{R}$ $(i \in \mathscr{I}, j=1,2, \ldots, N)$, and $c_{i}:=\left(c_{i, j}\right)_{j=1}^{N} \in \mathbb{R}^{N}(i \in \mathscr{I})$ with $c_{i, j}>0$. Then,

$$
\text { minimize } f(x):=\sum_{i \in \mathscr{I}} f_{i}(x) \text { subject to } x \in X:=\bigcap_{i \in \mathscr{I}} \operatorname{Fix}\left(Q_{i}\right)=\bigcap_{i \in \mathscr{I}} \operatorname{lev}_{\leq 0} g_{i},
$$

where $f_{i}: \mathbb{R}^{N} \rightarrow \mathbb{R}$ and $Q_{i}: \mathbb{R}^{N} \rightarrow \mathbb{R}^{N}$ are defined for all $x:=\left(x_{j}\right)_{j=1}^{N} \in \mathbb{R}^{N}$ by

$$
f_{i}(x):=\sum_{j=1}^{N} a_{i, j}\left|x_{j}-b_{i, j}\right|
$$

and

$$
Q_{i}(x):= \begin{cases}x-\frac{g_{i}(x)}{\left\|z_{i}(x)\right\|^{2}} z_{i}(x), & \text { if } g_{i}(x)>0, \\ x, & \text { if } x \in \operatorname{lev}_{\leq 0} g_{i}:=\left\{x \in \mathbb{R}^{N}: g_{i}(x) \leq 0\right\}\end{cases}
$$

$g_{i}: \mathbb{R}^{N} \rightarrow \mathbb{R}$ is defined for all $x \in \mathbb{R}^{N}$ by

$$
g_{i}(x):= \begin{cases}\left\langle c_{i}, x\right\rangle+d_{i}, & \text { if }\left\langle c_{i}, x\right\rangle>-d_{i}, \\ 0, & \text { otherwise }\end{cases}
$$


and $z_{i}(x)$ is any vector in $\partial g_{i}(x)$. The above mapping $Q_{i}$ is called the subgradient projection related to $g_{i}$. $Q_{i}$ satisfies quasi-firm nonexpnasivity, and Id $-Q_{i}$ satisfies the demiclosedness condition [1, Lemma 3.1].

The experiment was conducted on a MacBook Air (13-inch, 2017) with a $1.8 \mathrm{GHz}$ Intel (R) Core (TM) i5 CPU processor, 8 GB, $1600 \mathrm{MHz}$ DDR3 memory, and Mac OS Catalina (Version 10.15) operating system. PSM, ISM, and Algorithm 1 were written in Python 3.7.4 with the NumPy 1.17.2 package. We set $I=256$ and $N=1000$ and randomly chose $a_{i, j} \in(0,100]$, $b_{i, j} \in[-100,100), d_{i} \in[-1,0)$, and $c_{i, j} \in[-0.5,0.5)$. The stopping condition was $n=10000$. The step sizes were as follows:

$$
\begin{aligned}
& \text { Constant step sizes: } \gamma_{n}:=10^{-1}, 10^{-3}, \\
& \text { Diminishing step sizes: } \gamma_{n}:=\frac{10^{-1}}{n+1}, \frac{10^{-3}}{n+1} \text {. }
\end{aligned}
$$

The performance measures were as follows: for $n \in \mathbb{N}$,

$$
F_{n}:=\frac{1}{10} \sum_{s=1}^{10} \sum_{i \in \mathscr{I}} f_{i}\left(x_{n}(s)\right)
$$

and

$$
D_{n}:=\frac{1}{10} \sum_{s=1}^{10} \sum_{i \in \mathscr{I}}\left\|x_{n}(s)-Q_{i}\left(x_{n}(s)\right)\right\|,
$$

where $\left(x_{n}(s)\right)_{n \in \mathbb{N}}$ is the sequence generated by each of the three algorithms with the randomly chosen initial point $x_{0}(s) \in[0,1)^{N}(s=1,2, \ldots, 10)$. If $\left(D_{n}\right)_{n \in \mathbb{N}}$ converges to 0 , the algorithms converge to a point in $X$.

Figure 1 shows that the algorithms with $\gamma_{n}=\lambda_{n}=10^{-1}$ did not converge to a point in $X$. Figure 2 indicates that, although the values of $D_{10000}$ generated by the algorithms with $\gamma_{n}=\lambda_{n}=$ $10^{-3}$ were less than those generated by the algorithms with $\gamma_{n}=\lambda_{n}=10^{-1}$, the algorithms with $\gamma_{n}=\lambda_{n}=10^{-3}$ did not converge to a point in $X$. These results imply that it would be difficult to set an appropriate constant step size in advance.

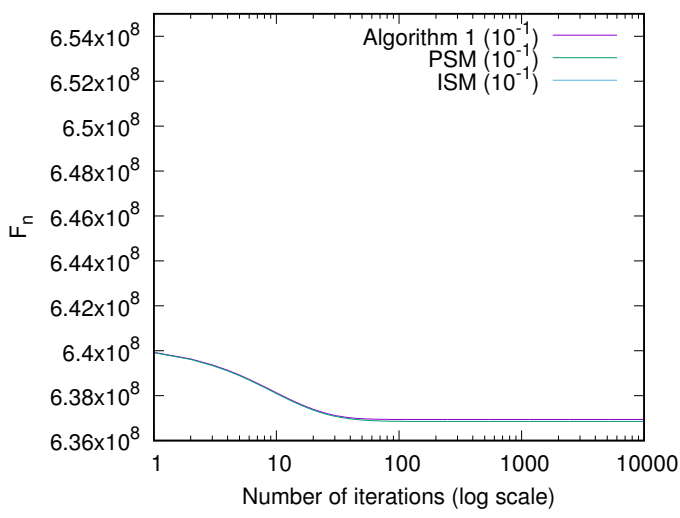

(A) Evaluation of $F_{n}$

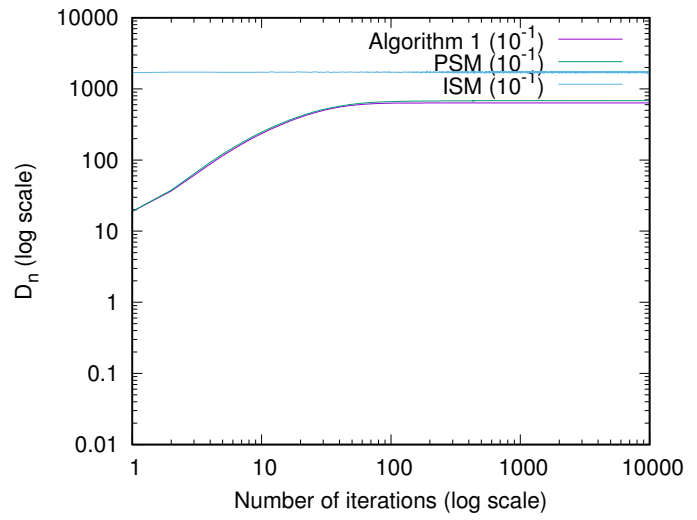

(B) Evaluation of $D_{n}$

FIgURE 1. Behaviors of $F_{n}$ and $D_{n}$ for Algorithm 1, PSM, and ISM with $\gamma_{n}=$ $\lambda_{n}=10^{-1}$ 


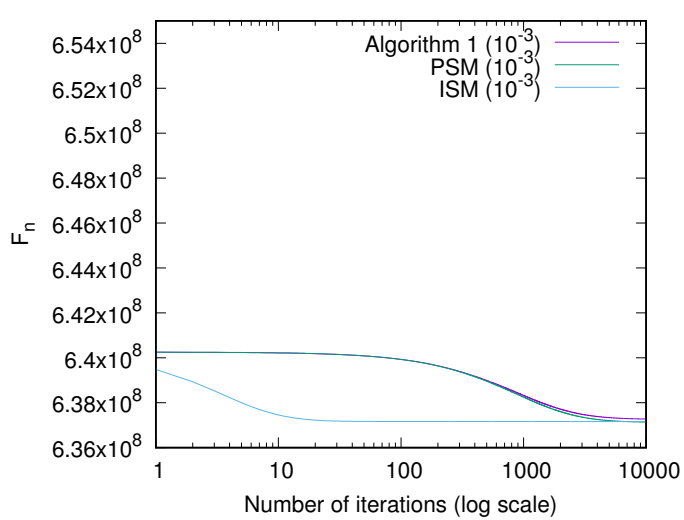

(A) Evaluation of $F_{n}$

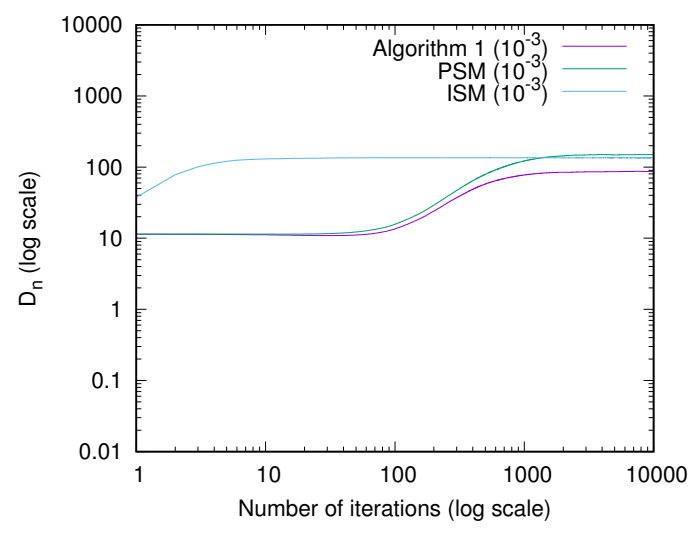

(B) Evaluation of $D_{n}$

FIGURE 2. Behaviors of $F_{n}$ and $D_{n}$ for Algorithm 1, PSM, and ISM with $\gamma_{n}=$ $\lambda_{n}=10^{-3}$

Meanwhile, Figures 3 and 4 show that Algorithm 1 with diminishing step sizes $\gamma_{n}=10^{-1} /(n+$ $1), 10^{-3} /(n+1)$ converged to a point in $X$, as guaranteed by Theorem 3.2. These figures also show that $F_{n}$ remains stable. Accordingly, from Theorem 3.2, Algorithm 1 converged to a solution of problem (4.1). Figures 3 and 4 also indicate that Algorithm 1 performs comparably to PSM and ISM.

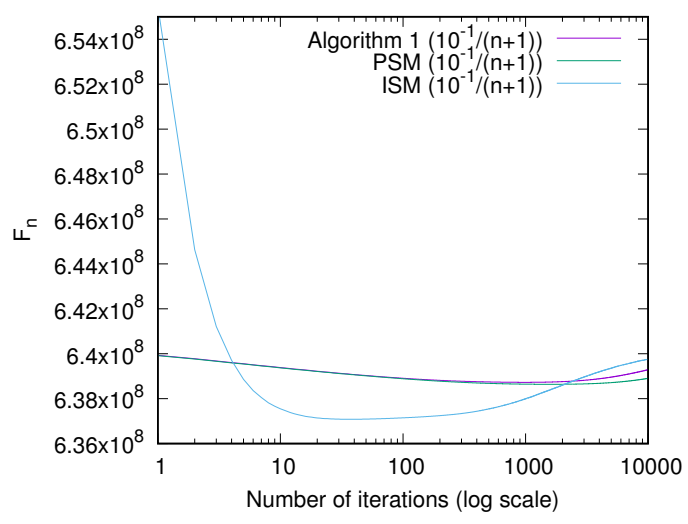

(A) Evaluation of $F_{n}$

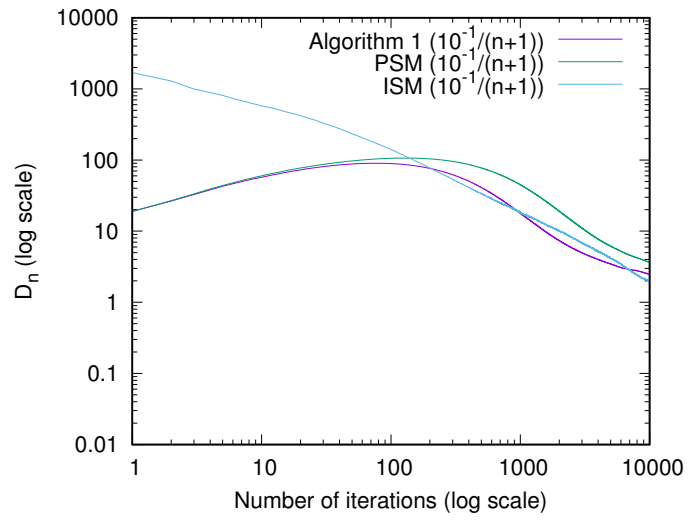

(B) Evaluation of $D_{n}$

FIGURE 3. Behaviors of $F_{n}$ and $D_{n}$ for Algorithm 1, PSM, and ISM with $\gamma_{n}=$ $\lambda_{n}=10^{-1} /(n+1)$ 


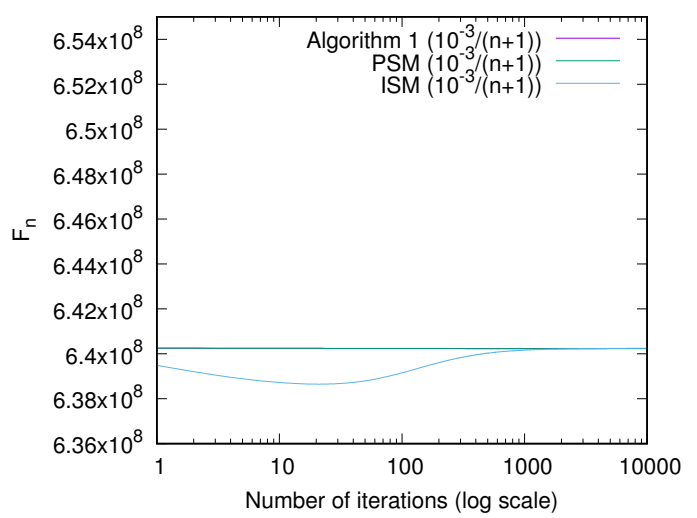

(A) Evaluation of $F_{n}$

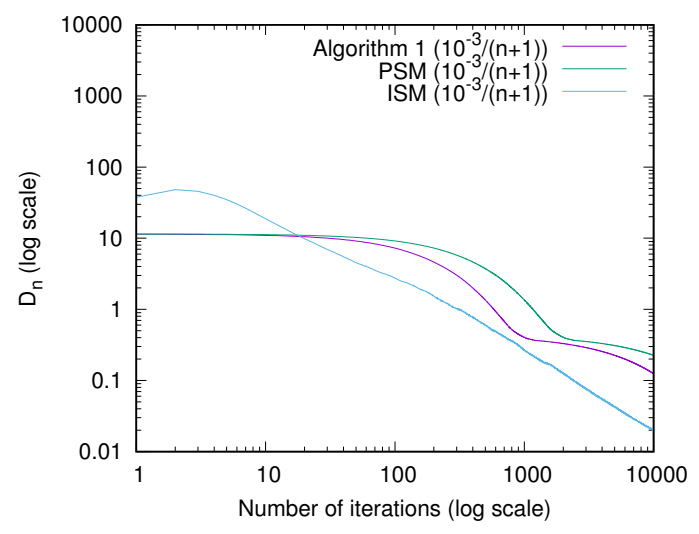

(B) Evaluation of $D_{n}$

FIgURE 4. Behaviors of $F_{n}$ and $D_{n}$ for Algorithm 1, PSM, and ISM with $\gamma_{n}=$ $\lambda_{n}=10^{-3} /(n+1)$

\section{The Conclusion}

This paper presented a parallel proximal method for solving the minimization problem of the sum of convex functions over the intersection of fixed point sets of quasi-nonexpansive mappings in a real Hilbert space. It also provided convergence and convergence-rate analyses. Numerical comparisons showed that the performance of the algorithm is almost the same as those of the existing methods.

\section{Acknowledgments}

The authors would like to thank Professor Xiaolong Qin for giving us a chance to submit our paper to this journal. This work was supported by JSPS KAKENHI Grant Numbers, JP18K11184 and JP17J09220.

\section{REFERENCES}

[1] H. H. Bauschke, J. Chen, A projection method for approximating fixed points of quasinonexpansive mappings without the usual demiclosedness condition, J. Nonlinear Convex Anal. 15 (2014), 129-135.

[2] H. H. Bauschke, P. L. Combettes, Convex Analysis and Monotone Operator Theory in Hilbert Spaces, (2011), Springer, New York.

[3] Y. Hayashi, H. Iiduka, Optimality and convergence for convex ensemble learning with sparsity and diversity based on fixed point optimization, Neurocomputing 273 (2018), 367-372.

[4] H. Iiduka, Fixed point optimization algorithms for distributed optimization in networked systems, SIAM J. Optim. 23 (2013), 1-26.

[5] H. Iiduka, Parallel computing subgradient method for nonsmooth convex optimization over the intersection of fixed point sets of nonexpansive mappings, Fixed Point Theory Appl. 2015 (2015), Article ID 72.

[6] H. Iiduka, Incremental subgradient method for nonsmooth convex optimization with fixed point constraints, Optim. Method Softw. 31 (2016), 931-951.

[7] H. Iiduka, Convergence analysis of iterative methods for nonsmooth convex optimization over fixed point sets of quasi-nonexpansive mappings, Math. Program. 159 (2016), 509-538.

[8] H. Iiduka, Proximal point algorithms for nonsmooth convex optimization with fixed point constraints, Eur. J. Oper. Res. 253 (2016), 503-513.

[9] H. Iiduka, Stochastic fixed point optimization algorithm for classifier ensemble, IEEE Trans. Cybernetics (2019). 
[10] H. Iiduka, Stochastic approximation methods using diagonal positive definite matrices for convex optimization with fixed point constraints, submitted.

[11] H. Iiduka, K. Hishinuma, Acceleration method combining broadcast and incremental distributed optimization algorithms, SIAM J. Optim. 24 (2014), 1840-1863.

[12] P. E. Maingé, The viscosity approximation process for quasi-nonexpansive mappings in Hilbert spaces, Comput. Math. Appl. 59 (2010), 74-79.

[13] G. J. Minty, A theorem on maximal monotonic sets in Hilbert space, J. Math. Anal. Appl. 11 (1965), 434-439.

[14] J. J. Moreau, Fonctions convexes duales et points proximaux dans un espace hilbertien, C. R. Acad. Sci. Paris Sér. A Math. 255 (1962), 2897-2899.

[15] Z. Opial, Weak convergence of the sequence of successive approximation for nonexpansive mappings, Bull. Amer. Math. Soc. 73 (1967), 591-597.

[16] K. Sakurai, T. Jimba, H. Iiduka, Iterative methods for parallel convex optimization with fixed point constraints, J. Nonlinear Var. Anal. 3 (2019), 115-126. 